\title{
CRABGRASS, MEASLES, AND GYPSY MOTHS: AN INTRODUCTION TO MODERN PROBABILITY
}

\section{RICHARD DURRETT}

This paper is based on a talk given at the Annual Meeting of the American Mathematical Society in San Antonio, Texas, January 21-24, 1978. The subject is interacting particle systems and the aim of the paper, like that of the talk, is to explain some of the results in this area to someone with no knowledge of probability theory except for an understanding of what it means to flip a coin with probability $p$ of heads. More than this is needed for some of the proofs given below, so a short appendix (three paragraphs) is provided which explains some of the concepts which may be unfamiliar.

In what follows we will discuss five models. The last three are hinted at in the title. The first two (Richardson's model and percolation) are related systems which are of interest in their own right, and will set the stage for explaining the other processes. Along the way the reader will encounter firstpassage percolation, the subadditive ergodic theorem, branching processes, and large deviations, and will see how interacting particle systems can be used to study nonlinear PDE's.

1. Richardson's model. In this model the state at time $n$ is $\xi_{n} \subset Z^{d}$. When considering this process as a model of the spread of a biological population, we think of the points of $\xi_{n}$ as being "occupied". At other times when we think of Richardson's model as describing the spread of an infection through an orchard of trees, we will call the points in $\xi_{n}$ "infected". Both interpretations are common in the literature and we will use both below as convenience dictates. Using the first interpretation, the evolution of the process may be described as follows:

If $x \in \xi_{n}$ then $x \in \xi_{n+1}$.

If $x \notin \xi_{n}$ then $\mathrm{P}\left(x \notin \xi_{n+1} \mid \xi_{n}\right)=(1-p) \#$ of occupied neighbors.

The first rule says there are no deaths. To explain the second rule we begin with the left-hand side. It says: "The probability that $x$ is not in $\xi_{n+1}$ given that $\xi_{n}$ is the state at time $n$." On the right-hand side, the neighbors of $x$ are the $2 d$ points with $\|x-y\|_{1}=1$ (where $\|x-y\|_{1}=\left|x_{1}-y_{1}\right|+\cdots+\left|x_{d}-y_{d}\right|$ ). In words, the rule says each occupied neighbor independently sends a particle to $x$ with probability $p$, so the probability they all fail to put a particle at $x$ is given by the right-hand side. The reader should note that the state at time $n$ is a subset of $Z^{d}$, i.e. each site is occupied by 1 or 0 particles, so if two neighbors simultaneously make the site occupied only one particle results.

Received by the editors April 13, 1987.

1980 Mathematics Subject Classification (1985 Revision). Primary 60K35.

Research partially supported by the National Science Foundation and by the Army Research Office through the Mathematical Sciences Institute at Cornell. 
Let $\xi_{n}^{0}$ be the state at time $n$ when initially only 0 is occupied. In Richardson's model $\xi_{n}^{0}$ grows and eventually contains all of $Z^{d}$, so attention focuses on how $\xi_{n}^{0}$ grows. The main result says the set of occupied sites has a limiting shape:

(1) THEOREM. There is a convex set $D$ so that for any $\varepsilon>0$ we have

$$
n(1-\varepsilon) D \cap Z^{d} \subset \xi_{n}^{0} \subset n(1+\varepsilon) D
$$

for all $n$ sufficiently large.

Loosely speaking $\xi_{n}^{0}$ looks like $n D \cap Z^{d}$ when $n$ is large. To get a feel for this result and how it is proved we begin with the trivial case $d=1$. In this situation if we let $t_{k}=\inf \left\{n: k \in \xi_{n}^{0}\right\}$ be the first time $k$ is infected, then it is easy to see that

$$
\mathrm{P}\left(t_{1}>m\right)=(1-p)^{m} \quad \text { for } m=0,1, \ldots,
$$

i.e., $t_{1}$ has a geometric distribution. A little more thought shows that $t_{2}-t_{1}$, $t_{3}-t_{2}, \ldots$ are independent random variables which have the same distribution as $t_{1}$, so the strong law of large numbers can be applied to conclude that with probability one

$$
t_{k} / k \text { converges to } \mathrm{E} t_{1} \text { as } k \rightarrow \infty,
$$

where $E$ denotes expected value and

$$
\mathrm{E} t_{1}=\sum_{m=1}^{\infty} m \mathrm{P}\left(t_{1}=m\right)=1 / p .
$$

Since $\left\{k \in \xi_{n}^{0}\right\}=\left\{t_{k} \leq n\right\}$, a little arithmetic shows that Theorem 1 holds with $D=[-p, p]$.

The simple argument above breaks down in $d>1$ because there is more than one way for the infection to spread from 0 to $x$, and a new approach is needed. We begin by reformulating the model to show that it is a special case of "first-passage percolation". Consider $Z^{d}$ as a graph with edges connecting each pair of points $x, y$ with $\|x-y\|_{1}=1$ and assign each edge an independent random variable $\tau(x, y)=\tau(y, x)$ with a geometric distribution. (When the geometric distribution is replaced by a general distribution the resulting model is called first-passage percolation.) If the infection reaches $x$ first then $\tau(x, y)$ is the amount of time that elapses before the particle at $x$ tries to infect the one at $y$. From the interpretation of $\tau(x, y)$ it should be clear that if $x_{0}=0$, $x_{1}, \ldots, x_{n}=y$ is a path from 0 to $y$ (i.e., $\left\|x_{i-1}-x_{i}\right\|_{1}=1$ for $i=1, \ldots, n$ ) then the "travel time" for this path, $\tau\left(x_{0}, x_{1}\right)+\cdots+\tau\left(x_{n-1}, x_{n}\right)$, gives an upper bound for the time it takes the infection to spread from 0 to $y$. A little more thought reveals that the first infection time

$$
t(y)=\inf \left\{n: y \in \xi_{n}^{0}\right\}
$$

is the infimum of the travel times over all paths from 0 to $y$.

In studying $t(y)$, it is useful to consider $t(x, y)=$ the minimum travel time for paths from $x$ to $y$ because this family of variables has the subadditivity property:

$$
t(x, y)+t(y, z) \geq t(x, z)
$$


(paths from $x$ to $y$ and from $y$ to $z$ can be combined to give a path from $x$ to $z$ but there are other ways to get from $x$ to $z$ ). (2) is called subadditivity. When $=$ holds in (2), as it did in $d=1, t(0, k)$ is a sum of $k$ independent random variables and the strong law of large numbers implies that with probability one

$$
t(0, k) / k \rightarrow \mathrm{E} t(0,1) \quad \text { as } k \rightarrow \infty .
$$

Somewhat remarkably, almost the same conclusion holds under (2):

(3) Let $t_{k}=t(0, k x)$ where $x \in Z^{d}$. With probability one

$$
t_{k} / k \rightarrow \inf _{j} \mathrm{E} t_{j} / j \quad \text { as } k \rightarrow \infty
$$

The first step in the proof is a nice exercise for an undergraduate analysis class: if $a_{k}=\mathrm{E} t_{k}$ then (1) implies $a_{j}+a_{k-j} \geq a_{k}$ and it follows from this that

$$
a_{k} / k \rightarrow \inf _{j} a_{j} / j \quad \text { as } k \rightarrow \infty .
$$

The rest of the proof is too involved to give here. A nice proof can be found in Liggett (1985a). To motivate the reader to look up this paper we would like to point out that the result proved there is a generalization of Kingman's (1973) subadditive ergodic theorem, which is in turn a very useful generalization of Birkhoff's ergodic theorem.

(3) gives a limiting result for the passage time to $(k z, 0)$ when $z \in Z^{d}$. If we extend the definition of the first-passage time to $x \in R^{2}$ by setting $t(x)=\inf \left\{n:[x] \in \xi_{n}^{0}\right\}$, where $[x]$ is the closest point in $Z^{2}$ to $x$ (with some convention for breaking ties) then an easy argument shows

(4) There is a constant $c(x)$ so that with probability one

$$
t(k x) / k \rightarrow c(x) \text {. }
$$

The last result gives radial limits for the first-passage times. With a little work this conclusion can be improved to show that (1) holds with $D=\{x: c(x) \leq 1\}$. The key step is to show that there is a $\delta>0$ so that $\mathrm{P}\left(\xi_{n}^{0} \supset\left\{x:\|x\|_{1} \leq n \delta\right\}\right) \rightarrow 1$. Once this is established a simple covering argument does the rest.

The expression for the limiting constant in (3) is mathematically nice because the infimum exists and is nonnegative and finite. It does not, however, lend itself well to computation (except of course for upper bounds), and very little is known about the limit set $D$ in $d>1$. One can, of course, make the trivial observations that $D$ has the same symmetry as $Z^{d}$, contains $\left\{x:\|x\|_{1} \leq p\right\}$, and is contained in $\left\{x:\|x\|_{1} \leq 1\right\}$, the limit when $p=1$. The only nontrivial information about $D$ is that in $d=2$ it has a "flat edge" when $p$ is close to 1, i.e. $D \cap\left\{x: x_{1}+x_{2}=1\right\}$ is an interval. See Durrett and Liggett (1981) for a proof.

NOTES. For more about first-passage percolation see Smythe and Wierman (1978), Cox and Durrett (1981), and Kesten (1986) or (1987b). An interesting higher-dimensional generalization which is an active topic of current research is treated in Kesten (1987a). 
2. Percolation. Percolation is one of the simplest systems which exhibits a "phase transition". In this model we again consider $Z^{d}$ as a graph with edges connecting each pair of points $x, y$ with $\|x-y\|_{1}=1$, but this time the edges (which we will call bonds) are designated as open or closed with probabilities $p$ and $1-p$ respectively, and the choices are made independently for each bond. We think of the open bonds as being air spaces which are large enough to permit the passage of a fluid. With this in mind we define the cluster containing $0: C_{0}=\{x: 0 \rightarrow x\}$, where $0 \rightarrow x$ is short for "there is a path of open bonds from 0 to $x$ " (and "path" is as defined in the previous section). $C_{0}$ is the set of sites which will become wet if there is a source of fluid at 0 . If $C_{0}$ is infinite then we say percolation occurs. The probability of percolation is a nondecreasing function of $p$, so it is natural to define the critical probability

$$
p_{c}=\inf \left\{p: \mathrm{P}\left(\left|C_{0}\right|=\infty\right)>0\right\} .
$$

In $d=1$ the percolation problem is trivial. $p_{c}=1$ because if $p<1$ then moving in either direction from 0 one will eventually encounter a closed bond and not be able to go further. In what follows then we will confine our attention to $d \geq 2$. The first thing to be proved is that $p_{c}$ is nontrivial, i.e., $0<p_{c}<1$. To prove the lower bound we begin by observing that if there is a path from 0 to $x$ then there is a self-avoiding path, i.e., one which visits any point at most once. The number of self-avoiding paths of length $n$ is $\leq 2 d(2 d-1)^{n-1}$ (the first step may be in any direction but after that we are forbidden to go back where we came from.) The probability that such a path is open is $p^{n}$ so the probability of having an open self-avoiding path of length $\geq N$ is at most

$$
\sum_{n=N}^{\infty} 2 d(2 d-1)^{n-1} p^{n}
$$

If $p<1 /(2 d-1)$ then the last sum $\rightarrow 0$ as $N \rightarrow \infty$, so $\mathrm{P}\left(\left|C_{0}\right|=\infty\right)=0$ and $p_{c} \geq 1 /(2 d-1)$.

To prove $p_{c}<1$ it suffices to consider the case $d=2$ since the critical value is a decreasing function of the dimension. Let

$$
W=\bigcup_{x \in C_{0}} x+[-1 / 2,1 / 2]^{2} .
$$

$W$ stands for wet region. In words, we have replaced each point in $C_{0}$ by a square of side 1 centered at the point so the result is a solid blob. If $C_{0}$ is finite, let $\Gamma$ be the boundary of the unbounded component of the open set $R^{2}-W$. $\Gamma$ is called the contour associated with $C_{0}$. If we let $Y^{2}=(1 / 2,1 / 2)+Z^{2}$ and consider $Y^{2}$ as a graph with edges connecting points $x, y \in Y^{2}$ with $\|x-y\|_{1}=1$ then drawing a picture (see Figure 1) one sees that $\Gamma$ is a union of edges on the graph $Y^{2}$ and each edge in $\Gamma$ crosses a closed bond on $Z^{2}$.

If the contour has length $n$ (i.e., it consists of $n$ edges) then the probability all the edges it crosses are closed is $(1-p)^{n}$. The contour is automatically self-avoiding and since it surrounds 0 , it contains some edge of the form $((k+1 / 2,-1 / 2),(k+1 / 2,1 / 2))$ where $0 \leq k<n / 2$. Combining the last two observations we see that the number of contours of length $n$ is less than $n 3^{n-1}$ 


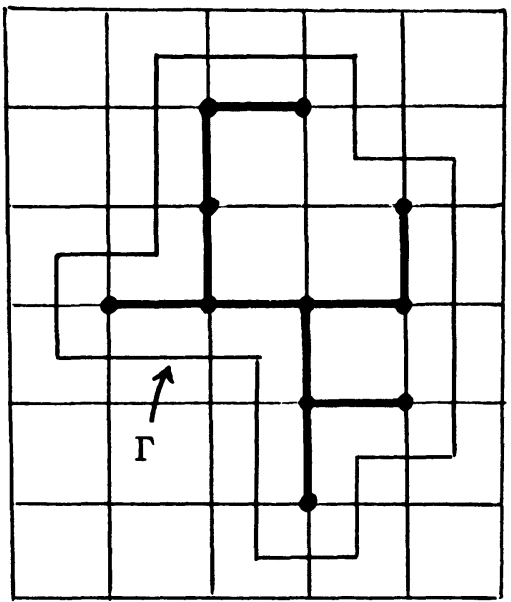

FIGURE 1

and that the probability of a contour of length greater than $N$ is at most

$$
\sum_{n=N}^{\infty} n 3^{n-1}(1-p)^{n}
$$

The shortest possible contour has length 4 so if $p$ is close to 1 the sum is less than 1 and there is positive probability that no contour exists (i.e., percolation occurs). To get a better bound on $p_{c}$ we observe that if $p>2 / 3$ then the last quantity goes to 0 as $N \rightarrow \infty$. To see that this is enough to show there is positive probability of percolation pick $N=4(2 M+1)$ to make the sum $<1$ and observe that if by a miracle all the bonds in $[-M, M]^{2}$ are open then the shortest possible contour around $C_{0}$ has length $N$ and given that the miracle occurs the probability that such a contour will exist is $<1$.

The last two results show that in $d=2$ we have $1 / 3 \leq p_{c} \leq 2 / 3$, and if one is good at leaping to conclusions one can guess that the answer is the midpoint of this interval: $p_{c}=1 / 2$. The first step in proving this was taken by Harris in 1960 when he showed $p_{c} \geq 1 / 2$. Four years later two physicists, Sykes and Essam, showed that if one assumed that the function

$$
f(p)=\sum_{n=1}^{\infty} n^{-1} \mathrm{P}_{p}\left(\left|C_{0}\right|=n\right)
$$

(the subscript on $\mathrm{P}$ indicating that bonds are open with probability $p$ ) which gives "the number of clusters per unit volume" has a singularity at $p=p_{c}$ but is otherwise smooth, then $p_{c}=1 / 2$, and for percolation on triangular and hexagonal lattices in $d=2$ one has $p_{c}=2 \sin (\pi / 18)$ [the unique root of $3 p-p^{3}=1$ in $\left.(0,1)\right]$ and $p_{c}=1-2 \sin (\pi / 18)$ respectively.

This state of affairs existed for over 15 years until Kesten showed in 1980 that $p_{c}=1 / 2$ and shortly thereafter Wierman generalized Kesten's proof to show that the numbers given above for the triangular and hexagonal lattice were also correct. The proof of Kesten's result is a somewhat lengthy story 
but it contains techniques which are important for what follows so we will give the main steps. A second motivation for describing the argument is that the proof given below is simpler in several respects than the original and although these simplifications are "widely known" they have not been explained in print. Even with the simplifications the proof is still messy so if the reader gets bored or confused he can safely skip ahead after finishing the proof of $p_{c} \geq 1 / 2$.

The keys to our proof are the idea of a sponge crossing and a "duality" for planar percolation problems hinted at in the proof of $p_{c} \leq 2 / 3$. A sponge is a rectangle $[0, m] \times[0, n]$, and we say a left-to-right crossing occurs if there is a path of open bonds from $\{0\} \times[0, n]$ to $\{m\} \times[0, n]$. A little graph theory (due to Whitney in the 1930s) shows that either there is a left-to-right crossing of $[0, m] \times[0, n]$ on $Z^{2}$ or there is a path on the dual graph $Y^{2}$ (described above) from the top to bottom of $[1 / 2, m-1 / 2] \times[-1 / 2, n+1 / 2]$ which only crosses bonds on $Z^{2}$ which are closed, but not both. The last sentence becomes easier to say if we declare the bonds on $Y^{2}$ to be open (resp. closed) if the bonds on $Z^{2}$ which they cross are open (resp. closed). We will use this convention in what follows.

When $m=n+1$ the original sponge and the dual sponge are both $n+1$ units long and $n$ units wide, so if $p=1 / 2$ it follows from symmetry that the probability of a right-to-left crossing is $1 / 2$. The last observation is one of the keys to the proof that $p_{c} \geq 1 / 2$. The second is the following useful result which is usually referred to as Harris's inequality.

(1) Let $A$ and $B$ be increasing events-i.e., if $A$ occurs for some configuration of open and closed bonds then it occurs in every outcome with more open bonds. Then

$$
\mathrm{P}(A \cap B) \geq \mathrm{P}(A) \mathrm{P}(B)
$$

The existence of an open path from one point (or set of points) to another is the basic example of an increasing event. If $A$ and $B$ are both events of this type then the lemma says that if we are lucky enough to find one connection then it increases the probability of finding the other one.

(1) is easy to prove (see Kesten (1982), pp. 72-73) but, as the reader will see below, it is very useful. The third and final ingredient in our proof is the following weird and wonderful result from Russo (1981). Let $\rho_{m, n}$ be the probability of a left-to-right crossing of an $m \times n$ sponge.

$$
\rho_{3 L / 2, L} \geq\left(1-\left(1-\rho_{L, L}\right)^{1 / 2}\right)^{3} \text {. }
$$

As you can probably guess from looking at the right side, the proof is a little tricky. The square root comes from the fact that if $A_{1}$ and $A_{2}$ are two increasing events with $A=A_{1} \cup A_{2}$ and $\mathrm{P}\left(A_{1}\right)=\mathrm{P}\left(A_{2}\right)$ then

$$
\begin{aligned}
\left(1-\mathrm{P}\left(A_{1}\right)\right)^{2} & =1-\mathrm{P}\left(A_{1}\right)-\mathrm{P}\left(A_{2}\right)+\mathrm{P}\left(A_{1}\right) \mathrm{P}\left(A_{2}\right) & & \text { since } \mathrm{P}\left(A_{1}\right)=\mathrm{P}\left(A_{2}\right) \\
& \leq 1-\mathrm{P}\left(A_{1}\right)-\mathrm{P}\left(A_{2}\right)+\mathrm{P}\left(A_{1} \cap A_{2}\right) & & \text { by }(1) \\
& =1-\mathrm{P}(A) & & \text { since } A=A_{1} \cup A_{2}
\end{aligned}
$$

or, rearranging,

$$
\mathrm{P}\left(A_{1}\right) \geq 1-(1-\mathrm{P}(A))^{1 / 2} \text {. }
$$


The nice thing about the last inequality is that if $\mathrm{P}(A)$ is close to 1 then so is the lower bound on $\mathrm{P}\left(A_{1}\right)$, and that is much better than just saying $\mathrm{P}\left(A_{1}\right) \geq \mathrm{P}(A) / 2$.

In proving (2), we apply the last result to get crossings which begin or end in the top (or bottom) half of the square, and if one does this cleverly left-to-right crossings of $[0, L] \times[0, L]$ and $[L / 2,3 L, 2] \times[0, L]$ and a top-tobottom crossing of the second square can be combined to get a crossing of $[0,3 L / 2] \times[0, L]$. The proof is very slippery (there are two incorrect proofs in the literature) and we have nothing to add to Russo's treatment, so we will not confuse the reader by giving the details.

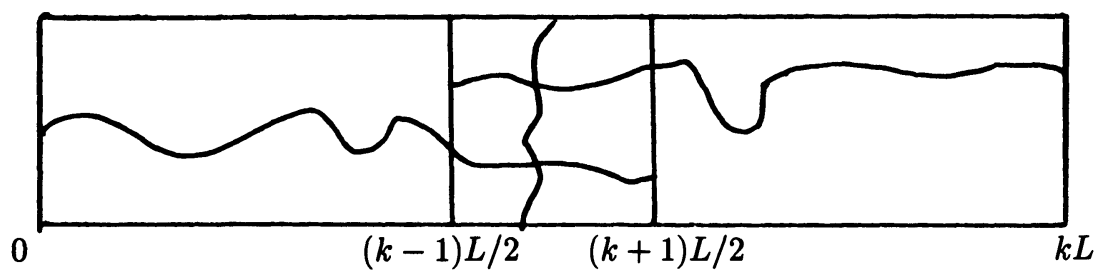

FIGURE 2

With (2) in hand the rest of the proof of $p_{c} \leq 1 / 2$ is smooth sailing. Drawing a picture (see Figure 2) shows

$$
\text { for } k \geq 1 \quad \rho_{k L, L} \geq\left(\rho_{(k+1) L / 2, L}\right)^{3} ;
$$

for if all three paths exist, the desired crossing occurs, and the three events are increasing so the probability of the intersection is bigger than the product of their probabilities. Using this result with $k=2$ and $k=3$ gives

$$
\rho_{2 L, L} \geq\left(\rho_{3 L / 2, L}\right)^{3}, \quad \rho_{3 L, L} \geq\left(\rho_{2 L, L}\right)^{3} .
$$

Combining the last two results with (2) shows that when $p=1 / 2$ we have

$$
\rho_{3 L, L} \geq\left(1-2^{-1 / 2}\right)^{27}=3.99 \times 10^{-15},
$$

a lower bound which is not very big but has the virtue of being independent of $L$.

When $p=1 / 2$ this bound applies equally well to crossing dual rectangles with closed paths. If we can cross $[n, 3 n] \times[-3 n, 3 n]$ and $[-3 n,-n] \times[-3 n, 3 n]$ from top to bottom, and cross $[-3 n, 3 n] \times[n, 3 n]$ and $[-3 n, 3 n] \times[-3 n,-n]$ from left to right with closed paths on the dual then we create a closed circuit of dual bonds which forces $C_{0}$ to be finite. The probability all four paths exist is by (4) and Harris's inequality at least

$$
\left(1-2^{-1 / 2}\right)^{108}=2.53 \times 10^{-58}>0 .
$$

This probability is ridiculously small, but taking $n=3^{k}$ gives an infinite sequence of disjoint square annuli in which the event can happen, so with probability 1 it will eventually happen and $C_{0}$ will be finite. 
Having shown that $p_{c} \geq 1 / 2$, the next step is to show that if $p>1 / 2$ then percolation occurs with positive probability. This is a two-step process: (i) first show there is an $\varepsilon_{0}>0$ so that if $\rho_{L, L}>1-\varepsilon_{0}$ then percolation has positive probability and then (ii) show that if $p>1 / 2$ then $\rho_{L, L} \rightarrow 1$ as $L \rightarrow \infty$. Given the developments above, the first step is easy. We start by observing that (3) could have been written as

$$
1-\rho_{k L, L} \leq 3\left(1-\rho_{(k+1) L / 2, L}\right)
$$

by observing that the probability some path in Figure 2 fails to exist is smaller than the right-hand side; a similar argument (see Figure 3) shows

$$
1-\rho_{4 L, L} \leq 5\left(1-\rho_{2 L, L}\right)
$$

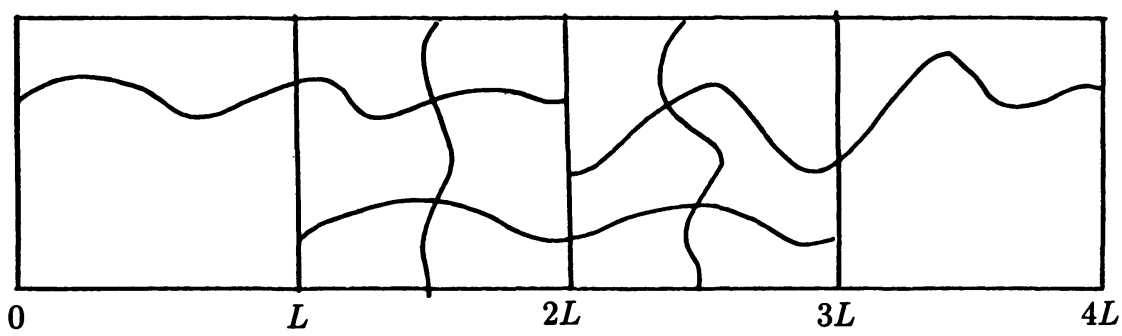

Figure 3

The second jab of our 1-2 punch is due to Aizenman, Chayes, Chayes, Frohlich, and Russo (1983):

$$
\rho_{4 L, 2 L} \geq 1-\left(1-\rho_{4 L, L}\right)^{2} .
$$

(7) is proved by placing two $4 L$ by $L$ rectangles next to each other and observing that we will cross the resulting $4 L$ by $2 L$ rectangle unless both $4 L$ by $L$ rectangles fail to be crossed and that those events are independent.

Combining the last two results gives

$$
\rho_{4 L, 2 L} \geq 1-25\left(1-\rho_{2 L, L}\right)^{2} .
$$

At first (8) may not look like much, but if we start with a small value, squaring is much more powerful than multiplying by 25 . To be less mystical, if $\rho_{2 L, L}=$ $1-\lambda / 25$ with $\lambda<1$ then (8) implies

$$
\begin{aligned}
& \rho_{4 L, 2 L} \geq 1-\lambda^{2} / 25 \\
& \rho_{8 L, 4 L} \geq 1-\lambda^{4} / 25
\end{aligned}
$$

and by induction

$$
\rho\left(2^{k} L, 2^{k-1} L\right) \geq 1-(1 / 25) \exp \left(2^{k-1} \log \lambda\right) .
$$

The last inequality shows that if $\rho_{2 L, L}$ is close enough to 1 then the sponge crossing probabilities converge to 1 exponentially fast. 
To get this scheme started we observe that if $\rho_{L, L}>1-\varepsilon$ then (2) implies

$$
\rho_{3 L / 2, L} \geq\left(1-\varepsilon^{1 / 2}\right)^{3}
$$

and (5) gives us

$$
\rho_{2 L, L} \geq 1-3\left(1-\rho_{3 L / 2, L}\right),
$$

so if $\varepsilon<\varepsilon_{0}$ then $\rho_{2 L, L}>.99$ and (9) holds with $\lambda=1 / 4$. To get from (9) to a positive probability of percolation pick $L$ so that $\rho_{L, L}>1-\varepsilon_{0}$, define boxes

$$
\begin{aligned}
B_{2 k-1} & =\left[0,2^{2 k-1} L\right] \times\left[0,2^{2 k-2} L\right], \\
B_{2 k} & =\left[0,2^{2 k-1} L\right] \times\left[0,2^{2 k} L\right],
\end{aligned}
$$

and observe that if we get left-to-right crossings of all the $B_{2 k-1}$ and topto-bottom crossings of all the $B_{2 k}$ then there is an infinite path starting on $\{0\} \times[0, L]$ (see Figure 4). The probability that we get all the paths we want and that in addition all the bonds on $\{0\} \times[0, L]$ are open is by Harris's inequality at least

$$
p^{L} \cdot \prod_{k=1}^{\infty}\left[1-(1 / 25) \exp \left(-2^{k-1} \log 4\right)\right]>0
$$

so we have demonstrated (i).

Last but not least we come to (ii). The key to the proof of this is Russo's formula:

(10) if $A$ is an increasing event then

$$
\frac{d}{d p} \mathrm{P}_{p}(A)=\mathrm{E}(\# \text { of pivotal bonds })
$$

where a bond is said to be pivotal if changing its state changes the occurrence of the event; i.e., when the bond is open $A$ occurs but if the bond is closed it does not.

To prove (ii) we apply this fact to $A_{L}=$ there is a left-to-right crossing of $[0, L+1] \times[0, L]$ with $L$ large, and show that if $p \geq 1 / 2$ and $\mathrm{P}_{p}\left(A_{L}\right)<1-\varepsilon_{0}$ (the magic constant in the proof of (i)) then the derivative is large. Since $\mathrm{P}_{1 / 2}\left(A_{L}\right)=1 / 2$, this fact implies that

$$
\inf \left\{p: \mathrm{P}_{p}\left(A_{L}\right)>1-\varepsilon_{0}\right\} \rightarrow 1 / 2,
$$

and in view of the proof of (i) it follows that $\rho_{L, L} \rightarrow 1$ for all $p>1 / 2$.

To complete the proof now we need to get our hands dirty and find a large number of pivotal bonds. The fact that this step is nontrivial can be proved by noting that Russo (1978) knew that large sponge-crossing probabilities implied percolation (and was aware of Russo's formula!), but he could not complete the proof, nor could anyone else, until Kesten came along and finished the problem.

Kesten's idea was to look at the lowest left-to-right crossing. The event that $\sigma$ is the lowest crossing is determined by the bonds which lie on or below $\sigma$, so if we condition on $\sigma$ being the lowest crossing the distribution of the bonds which lie above it is not affected by the conditioning. In this virgin territory which lies above $\sigma$ we look for a closed path on the dual which comes 


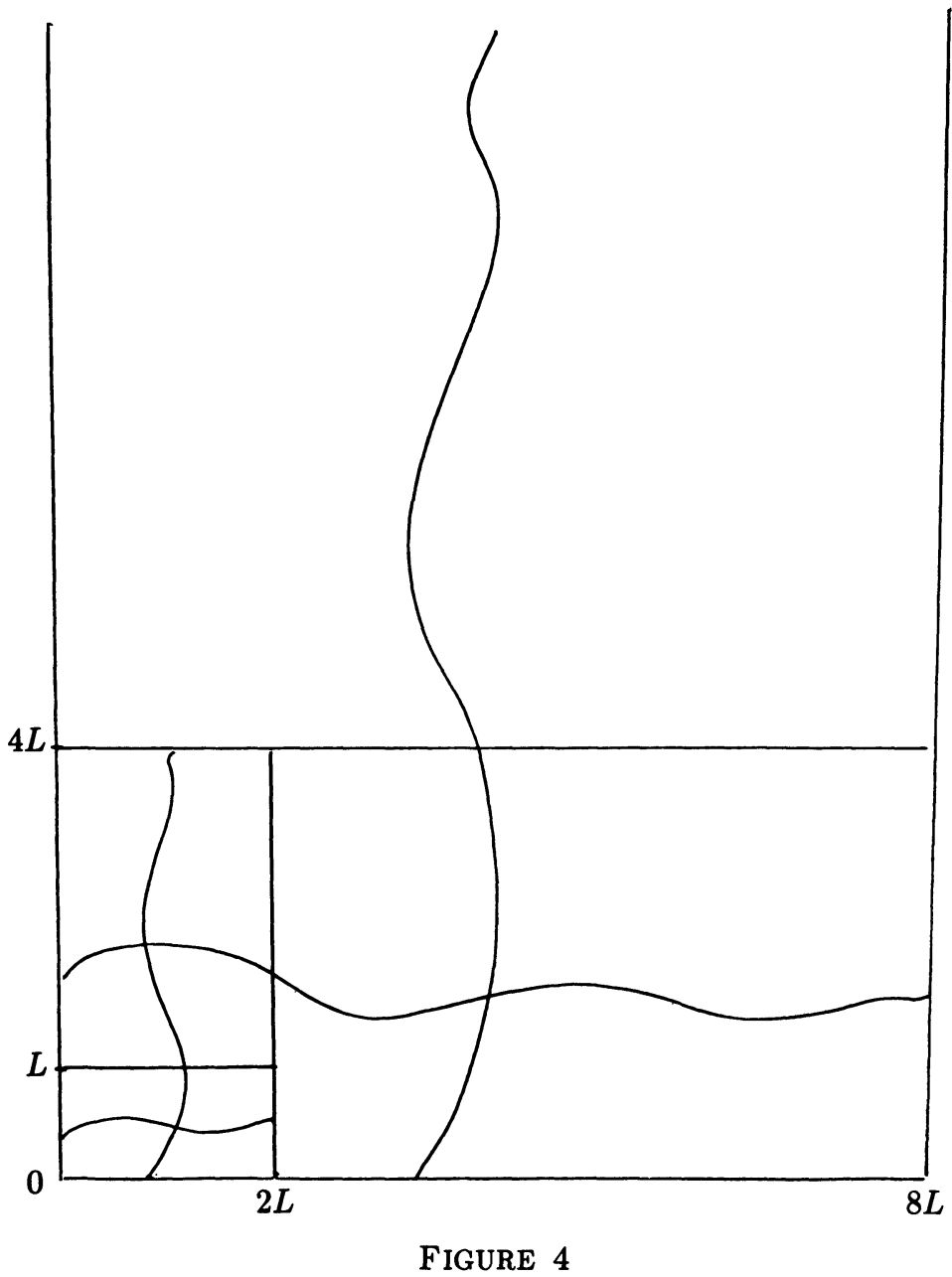

down and touches $\sigma$. If $\mathrm{P}_{p}\left(A_{L}\right) \leq 1-\varepsilon_{0}$ then the probability of finding one is at least $\varepsilon_{0}$ (the probability of a top-to-bottom dual crossing by closed bonds is $1-\mathrm{P}\left(A_{L}\right)$ and all we want to do is go down to $\left.\sigma\right)$. If there is a path on the dual from the top down to $\sigma$ call it $\omega$ (if there are more than one, pick the left-most one).

The bond at the point where $\omega$ touches $\sigma$ is pivotal since $\sigma$ is the lowest crossing and the presence of $\omega$ implies that every crossing must pass through this bond. In the region to the right of $\omega$ and above $\sigma$ (which is still virgin territory) we look for more closed crossings by introducing roughly $\log _{3} L$ disjoint square annuli centered at the point where $\omega$ touches $\sigma$ and looking for closed circuits in these annuli which go from $\omega$ to $\sigma$. Every circuit produces at least one pivotal bond and when $\mathrm{P}\left(A_{L}\right) \leq 1-\varepsilon_{0}$ there is a lower bound on the probability of these circuits, so the expected number is at least $\delta \log L$ for some $\delta>0$. 
Further details are left to the reader. Readers who want to see why Kesten is famous for his ability to compute things should look at Kesten (1981), where the construction above is sharpened to produce $L^{\delta}$ pivotal bonds. This improvement is needed if one wants to get nontrivial bounds on "critical exponents". Critical exponents are of central importance in the study of phase transitions but a little too complicated to discuss here.

NOTES. The classic reference for percolation is Kesten (1982) but this treatment is hard to read since it treats all planar percolation problems simultaneously. Wierman's (1982) survey and Kesten's (1987b) paper are more readable; the latter reference will fill you in on the current state of knowledge. For a treatment of a random surface problem which is the dual of three-dimensional percolation see Aizenman, Chayes, Chayes, Frohlich, and Russo (1983).

3. Measles. The next process can be used to model the spread of a disease or a forest fire. The state of the process at time $n$ is a function $\xi_{n}: Z^{2} \rightarrow\{1, i, 0\}$. The states $1, i$, and 0 have the following meanings in the two interpretations:

\begin{tabular}{lll} 
& fire & measles \\
\hline $\mathbf{1}$ & tree & healthy \\
$\boldsymbol{i}$ & on fire & infected \\
$\mathbf{0}$ & burnt & immune
\end{tabular}

We have chosen measles for the disease because in that case once you have had the disease you cannot have it again. With this or a forest fire in mind, the dynamics of the model can be described as follows:

If $\xi_{n}(x)=0$ then $\xi_{n+1}(x)=0$.

If $\xi_{n}(x)=i$ then $\xi_{n+1}(x)=0$.

If $\xi_{n}(x)=1$ then

$$
\begin{aligned}
& \mathrm{P}\left(\xi_{n+1}(x)=1 \mid \xi_{n}\right)=(1-p)^{\# \text { of infected neighbors }} \\
& \mathrm{P}\left(\xi_{n+1}(x)=i \mid \xi_{n}\right)=1-\mathrm{P}\left(\xi_{n+1}(x)=1 \mid \xi_{n}\right) .
\end{aligned}
$$

The reason for the first rule was explained above. In the second we have taken the unreasonable viewpoint that the disease always lasts for exactly one unit of time. This can be generalized considerably but we only consider the simplest case here. The third rule should be familiar from the Richardson's model: each infected neighbor independently tries to infect $x$ with probability $p$.

We will be interested in what happens when the initial state is given by: $\xi_{0}(0)=i$ and $\xi_{0}(x)=1$ for $x \neq 0$. Let $I_{n}=\left\{x: \xi_{n}(x)=i\right\}$ be the set of infected individuals at time $n$ and let $\Omega_{\infty}=\left\{I_{n} \neq \varnothing\right.$ for all $\left.n\right\}$ be the event that the infection does not die out. The probability of $\Omega_{\infty}$ is a nondecreasing function of $p$, so we define

$$
p_{c}=\inf \left\{p: \mathrm{P}_{p}\left(\Omega_{\infty}\right)>0\right\} .
$$

The first thing we will prove about this model is that $p_{c}=1 / 2$. Given the fact that $p_{c}$ was $1 / 2$ in the last section the reader has probably already leapt to the conclusion that this process has something to do with percolation. To 
spell out the connection we need to introduce a special construction of the process under consideration. At each point $x$ and for each of its neighbors $y$ we flip a coin with probability $p$ of heads to see if $x$ will try to infect $y$ at the one time $x$ is infected (if this ever occurs), and we draw an arrow from $x$ to $y$ if it will try. Let $C_{0}=\{x: 0 \rightarrow x\}$ where $0 \rightarrow x$ means there is a path of arrows from 0 to $x$. A little thought reveals that $C_{0}$ is the set of sites which will ever be infected and $\Omega_{\infty}=\left\{C_{0}\right.$ is infinite $\}$.

The percolation process described in the last paragraph is not the bond percolation model described in the last section since in that process we flip a coin for each pair of neighbors $x$ and $y$ to see if they are connected by a bond which can be traversed in either direction and here we use two different coins for the two oriented edges $(x, y)$ and $(y, x)$. Somewhat surprisingly it is equivalent in a very strong sense: if $S$ and $T$ are two subsets of $Z^{2}$ then the probability of a path from $S$ to $T$ is the same in the two models. The last fact is surprising, but once you realize that it is true for any graph, it can easily be verified by induction on the number of bonds in the graph (with a passage to the limit to handle $Z^{2}$ ).

The key to the proof is the observation that when we add a new edge $(a, b)$ to the undirected graph and two edges $(a, b)$ and $(b, a)$ to the corresponding directed graph then one of three things can happen: (i) there was already a connection from $S$ to $T$; (ii) there is no path from $S$ to $T$ but there is a path from $S$ to $a$ and from $b$ to $T$ so if the oriented edge $(a, b)$ is open there is a path from $S$ to $T$; (iii) the situation in (ii) occurs and in addition there is a path from $S$ to $b$ and from $a$ to $T$ so if $(a, b)$ is open in either direction there is a path from $S$ to $T$. At first it looks like case (iii) torpedoes the proof since the oriented model has two chances to make the connection while the unoriented model has one. A closer look, however, reveals case (iii) is a special case of (i): there is a path from $S$ to $b$ and from $b$ to $T$ so there is already a path from $S$ to $T$. With (iii) out of the way we are left with case (ii) in which each model has exactly one chance to make the desired connection and each will succeed with probability $p$.

With the equivalence just described in hand, the fact that $p_{c}=1 / 2$ follows from results in the last section. Let $S=\{0\}, T_{n}=\left\{x:\|x\|_{1}=n\right\}$ and let $n \rightarrow \infty$. The reader should note that this argument shows that the percolation probability is the same in the two models and the result above implies that sponge crossing probabilities have exactly the same values. The last observation makes the techniques used to study percolation available to treat the model under consideration. Returning to a theme of $\S 1$, we can use these techniques to prove a "shape theorem".

(1) THEOREM. Let $J_{n}=\left\{x: \xi_{n}(x)=0\right\}$ be the set of sites which are immune at time $n$. There is a convex set $D$ so that for all $\varepsilon>0$

$$
n(1-\varepsilon) D \cap C_{0} \subset J_{n} \subset n(1+\varepsilon) D
$$

for all $n$ sufficiently large. 
The key to the proof of this result is, as in $\S 1$, a subadditivity property of the first-infection times

$$
t(x)=\inf \left\{n: \xi_{n}(x)=i\right\}
$$

To introduce the subadditivity property we will again reformulate things in terms of first-passage percolation. Assign the oriented bond $(x, y)$ the value 1 if an arrow was drawn from $x$ to $y$ in the construction above, and assign $(x, y)$ the value $\infty$ if not. If we let $\tau(x, y)$ be the value assigned to $(x, y)$ then again $\tau(x, y)$ is the amount of time that will elapse between the time $x$ first becomes infected and the time it tries to infect $y$. From the interpretation of $\tau(x, y)$ and arguments in $\S 1$ it should be clear that the first-infection time $t(x)$ is the infimum of the travel times over all paths from 0 , and if we let $t(x, y)$ be the minimum passage time from $x$ to $y$ then

$$
t(x, y)+t(y, z) \geq t(x, z) .
$$

The infection times $t(x, y)$ are infinite with positive probability, so we cannot directly apply the subadditive ergodic theorem. To get around this difficulty let $p>1 / 2$ and observe that results in the last section imply that the probability of a left-to-right crossing of $[0,3 L] \times[0, L]$ goes to 1 exponentially fast as $L \rightarrow \infty$. If we can cross $[-3 n, 3 n] \times[n, 3 n]$ from left to right, $[n, 3 n] \times[-3 n, 3 n]$ from top to bottom, $[-3 n, 3 n] \times[-3 n,-n]$ from right to left, and $[-3 n,-n] \times[-3 n, 3 n]$ from bottom to top then we have a clockwise circuit of arrows surrounding 0 . Let $\Delta(0)$ be the smallest such circuit which is connected to $\infty$ : i.e., if $x \in \Delta(0)$ there are an infinite number of points $y$ with $x \rightarrow y$, where $x \rightarrow y$ means there is a path of arrows from $x$ to $y$. Using the construction we used in the last section to produce percolation when $p>1 / 2$, it is easy to show that $\Delta(0)$ exists and furthermore

$$
\mathrm{P}(\text { radius of } \Delta(0) \geq n) \leq C e^{-\gamma n}
$$

where $C, \gamma \in(0, \infty)$.

Our substitute for the passage times $t(x, y)$ are the times $s(x, y)=$ the minimum passage time from $\Delta(x)$ to $\Delta(y)$. The times $s(x, y)$ are not subadditive but if we let $u(x)$ be the number of arrows on or inside $\Delta(x)$ then

$$
s(x, y)+u(y)+s(y, z) \geq s(x, z) .
$$

Adding $u(z)$ to both sides and letting $r(x, y)=s(x, y)+u(y)$ then we get

$$
r(x, y)+r(y, z) \geq r(x, z)
$$

The $r(x, y)$ are always finite since if $x_{0}, x_{1}, \ldots, x_{n}$ is a path from $x$ to $y$ one can build a path from $\Delta(x)$ to $\Delta(y)$ from $\Delta\left(x_{0}\right) \cup \Delta\left(x_{1}\right) \cup \cdots \cup \Delta\left(x_{n}\right)$. The bound (3) on the size of the $\Delta\left(x_{i}\right)$ implies $\operatorname{Er}(x, y)<\infty$ (in fact $\mathrm{E}(r(x, y))^{k}<\infty$ for all $k$ ), so the subadditive ergodic theorem can be applied to conclude that if $r_{k}=r(0, k x)$ with $x \in Z^{2}$ then with probability one

$$
r_{k} / k \rightarrow \inf _{j} \mathrm{E} r_{j} / j .
$$

To get from this result to a limit theorem for the $t(x, y)$ we observe that if $t(0, k x)<\infty$

$$
r(0, k x)-u(k x)=s(0, k x) \leq t(0, k x) \leq u(0)+r(0, k x),
$$


$u(0) / k \rightarrow 0$ as $k \rightarrow \infty$. To estimate the other error we observe that for any $\varepsilon>0$

$$
\infty>\mathrm{E} u(0) / \varepsilon \geq \sum_{k=1}^{\infty} \mathrm{P}(u(0) / \varepsilon \geq k)=\sum_{k=1}^{\infty} \mathrm{P}(u(k x) \geq k \varepsilon)
$$

so

$$
\mathrm{P}(u(k x) / k \geq \varepsilon \text { for some } k \geq K) \rightarrow 0 \text { as } K \rightarrow \infty .
$$

If we let $c(x)$ be the right-hand side of (6) then we have shown

$$
\mathrm{P}(|t(0, k x)-k c(x)|<k \varepsilon, t(0, k x)<\infty) \rightarrow 0 .
$$

for all $\varepsilon>0$. As in $\S 1$, if we extend the definition of passage times to $x \in R^{2}$ by setting

$$
t(x)=\inf \left\{n: \xi_{n}([x])=i\right\},
$$

where $[x]$ is the closest point in $Z^{2}$ to $x$, then an easy argument shows that (7) remains valid and a little more work gives the conclusion stated in the theorem.

NOTES. The techniques used above are mostly from Cox and Durrett (1981). The proof of (1) will appear in Cox and Durrett (198?). That paper treats a more general model in which a site stays infected for an amount of time with distribution $F$ and while infected emits germs according to a Poisson process with rate $\alpha$, the germ landing at one of the four neighbors chosen at random. In the general model the bonds out of a point are not independent (they are positively correlated) so one does not have the equivalence used above and one must generalize the results of the last section.

4. Gypsy moths. In this process (officially called the contact process) and the next one, time is continuous, i.e. the process is defined for all $t \geq 0$. Otherwise, these processes are much like a version of Richardson's model in which occupied sites become vacant with positive probability. The state at time $t$ is $\xi_{t} \subset Z^{d}$. As before, we could think of $\xi_{t}$ as the set of "occupied" sites, but to be true to our title, we think of the points of $Z^{d}$ as trees, and the $\xi_{t}$ as indicating the trees infested by gypsy moths.

The system evolves according to the following rules:

If $x \in \xi_{t}, \mathrm{P}\left(x \notin \xi_{t+s} \mid \xi_{t}\right)=s+o(s)$.

If $x \notin \xi_{t}, \mathrm{P}\left(x \in \xi_{t+s} \mid \xi_{t}\right)=\lambda s$ (\# of occupied neighbors) $+o(s)$.

Here $o(s)$ means that the missing terms when divided by $s$ go to 0 as $s \rightarrow 0$. For readers familiar with Markov chains, the rules may be expressed as: particles die at rate one, and are born at vacant sites at rate $\lambda$ (\# of occupied neighbors).

If you are not familiar with Markov chains, that is not important: we will now construct the process from a graphical representation. For each $x$ let $\left\{T_{n}^{x}, n=1,2, \ldots\right\}$ be a Poisson process with rate 1 , i.e., if we let $T_{0}^{x}=0$ then $T_{n}^{x}-T_{n-1}^{x}$ are independent random variables which have

$$
\mathrm{P}\left(T_{n}^{x}-T_{n-1}^{x}>t\right)=e^{-t}
$$

At times $T_{n}^{x}, n=1,2, \ldots$, we write a $\delta$ (for death) to indicate that if $x$ was occupied at time $T_{n}^{x}$ it will become vacant. 
To take care of the births we introduce a Poisson process $\left\{T_{n}^{x, y}, n=\right.$ $1,2, \ldots\}$ with rate $\lambda$ for each pair of neighbors $x, y$, i.e., if we let $T_{0}^{x, y}=0$ then $T_{n}^{x, y}-T_{n-1}^{x, y}, n=1,2, \ldots$, are independent random variables which have

$$
\mathrm{P}\left(T_{n}^{x, y}-T_{n-1}^{x, y}>t\right)=e^{-\lambda t} .
$$

At times $T_{n}^{x, y}, n=1,2, \ldots$, we draw an arrow from $x$ to $y$ to indicate that if $x$ is occupied at time $T_{n}^{x, y}$ then $y$ will become occupied at that time (if it is not already occupied).

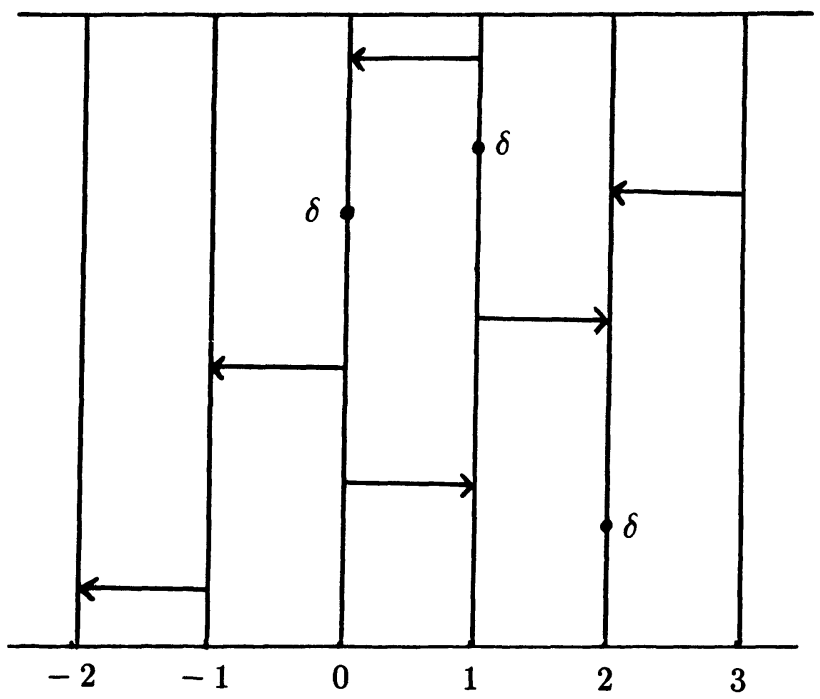

FIGURE 5

Given the "graphical representation" described above, we can construct the contact process in a manner which should remind the reader of the percolation processes discussed in the last two sections. (The reader should consult Figure 5 for help with the definitions.) We say there is a path from $(x, 0)$ to $(y, t)$ and write $(x, 0) \rightarrow(y, t)$ if there is a sequence of times $s_{0}=0<s_{1}<\cdots<$ $s_{n}<s_{n+1}=t$ and spatial locations $x_{0}=x, x_{1}, \ldots, x_{n}=y$ so that

(i) for $i=1,2, \ldots, n$ there is an arrow from $x_{i-1}$ to $x_{i}$ at time $s_{i}$, and

(ii) the vertical segments $\left\{x_{i}\right\} \times\left(s_{i}, s_{i+1}\right), i=0,1, \ldots, n$ do not contain any $\delta$ 's.

If there is a path from $(x, 0)$ to $(y, t)$ and $x$ was occupied at time 0 then $y$ will be occupied at time $t$. Given this we define the contact process starting from $\xi_{0}=A$ by

$$
\xi_{t}^{A}=\{y: \text { there is an } x \text { in } A \text { so that }(x, 0) \rightarrow(y, t)\}
$$

(in the realization drawn in Figure $5, \xi_{t}^{\{0\}}=\{-1,2\}, \xi_{t}^{\{1\}}=\{2\}$, and $\xi_{t}^{\{2\}}=$ $\varnothing)$.

From the descriptions above, it should be clear that the contact process is a continuous-time percolation process. With this analogy in mind, we let 
$\Omega_{\infty}=\left\{\xi_{t}^{0} \neq \varnothing\right.$ for all $\left.t\right\}$ (where $\xi_{t}^{0}$ is short for $\xi_{t}^{\{0\}}$ ) be the event that the process does not die out, starting from a single individual at 0 , and let

$$
\lambda_{c}=\inf \left\{\lambda: P\left(\Omega_{\infty}\right)>0\right\} .
$$

This time, in contrast to the last two sections, we do not know the value of $\lambda_{c}$, but in the study of interacting systems, this situation is typical and the last two models are the exceptions. Two results about $\lambda_{c}$ worth mentioning are: (i) It is easy to show $\lambda_{c} \geq 1 / 2 d$. (A particle with no occupied neighbors gives birth at rate $2 d \lambda$ and a particle with some occupied neighbors gives birth at a smaller rate. All particles die at rate 1 so if $2 d \lambda<1$ the process dies out.) (ii) A clever argument of Holley and Liggett (see §VI.1 in Liggett (1985b)) shows $\lambda_{c} \leq 2 / d$. The second bound is good in low dimensions and the first in high dimensions. Numerically $\lambda_{c}=1.65$ in $d=1, \lambda_{c}=0.41$ in $d=2$, and it has been shown that $2 d \lambda_{c} \rightarrow 1$ as $d \rightarrow \infty$.

In $\S \S 1$ and 3 we have seen that starting from a finite set the system expands linearly and has an asymptotic shape. This is true again here, but we will have to introduce a few concepts to state the limit result. We begin by considering what happens in the process $\xi_{t}^{1}$ starting from all sites occupied, i.e. $\xi_{0}^{1}=Z^{d}$. Now $Z^{d}$ is the largest state (in the partial order $\supset$ ), and the construction of the model described above has the property that if we use it to run two versions of the process $\xi_{t}^{A}$ and $\xi_{t}^{B}$ starting from initial states $A \supset B$, then we will have $\xi_{t}^{A} \supset \xi_{t}^{B}$ for all $t$. If we let $A=Z^{d}$ and $B=\xi_{s}^{1}$ in the last observation, then we see that $\xi_{t}^{1}$ is larger than $\xi_{t+s}^{1}$ in the sense that the two random sets can be constructed on the same space with $\xi_{t}^{1} \supset \xi_{t+s}^{1}$. Once one understands the last sentence, a simple argument shows that, as $t \rightarrow \infty, \xi_{t}^{1}$ decreases to a limit we call $\xi_{\infty}^{1}$, where the convergence occurs in the sense that

$$
\mathrm{P}\left(\xi_{t}^{1} \cap C \neq \varnothing\right) \downarrow \mathrm{P}\left(\xi_{\infty}^{1} \cap C \neq \varnothing\right) \quad \text { for all finite sets } C .
$$

It follows from Markov chain theory that $\xi_{\infty}^{1}$ is an equilibrium distribution for the process, i.e. if the initial state has this distribution, then this will be the distribution at all $t \geq 0$. If $\lambda<\lambda_{c}$ then $\xi_{\infty}^{1}$ is not interesting-it is $\varnothing$ with probability 1 -but if $\lambda>\lambda_{c}$ it is a nontrivial equilibrium distribution. The reader will note that $\lambda=\lambda_{c}$ has been left out in the last statement. Presumably this value falls under the first case [recall that the results of $\S 2$ show that there is no percolation when $p=p_{c}$ and the equivalence of models demonstrated in $\S 3$ shows that the forest fire dies out at the critical value], but this is a very difficult open problem.

With the equilibrium distribution introduced, we are now in a position to describe the limiting behavior starting from a finite set. Suppose we use the construction above to run two versions of the process, one starting from a finite set $A$ and the other starting from all of $Z^{d}$ occupied, and we call the two resulting processes $\xi_{t}^{A}$ and $\xi_{t}^{1}$. The "shape theorem" in this setting is:

(1) THEOREM. If $\lambda>\lambda_{c}^{*}$ then there is a convex set $D$ so that if $\xi_{t}^{A} \neq \varnothing$ for all $t$, then for any $\varepsilon>0$ we have

$$
\left(t(1-\varepsilon) D \cap \xi_{t}^{1}\right) \subset \xi_{t}^{A} \subset t(1+\varepsilon) D
$$

for all $t$ sufficiently large. 
The statement of the result is made contorted by the fact that $\xi_{t}^{A}$ may become $\varnothing$, in which case it stays $\varnothing$ for all time. The theorem tells us that when this does not occur $\xi_{t}^{A}$ looks roughly like $\xi_{\infty}^{1} \cap t D$, or, in words, it is a linearly growing "blob in equilibrium". Last but not least, we have $\lambda_{c}^{*}$ instead of $\lambda_{c}$ in the statement of the theorem. (Unfortunately this is not a typo!) To explain what $\lambda_{c}^{*}$ is and why we think it is equal to $\lambda_{c}$ will take some time. For the moment however this is not relevant. We will begin by discussing the case $d=1$, where the result is known to hold for all $\lambda>\lambda_{c}$.

Let $\xi_{t}^{-}$denote the contact process starting from $\xi_{0}^{-}=\{0,-1,-2, \ldots\}$ and let $r_{t}=\sup \xi_{t}^{-}$be the rightmost occupied site at time $t$. If we let $r_{s, t}=$ $\sup \left\{y-r_{s}:(x, s) \rightarrow(y, t)\right.$ for some $\left.x \leq r_{s}\right\}$ then a moment's thought reveals

$$
r_{s}+r_{s, t} \geq r_{t}
$$

so $r_{t}$ is subadditive, and it follows from the result quoted in $\S 1$ that with probability one as $t \rightarrow \infty$

$$
r_{t} / t \rightarrow \alpha(\lambda)=\inf _{s} \mathrm{E}\left(r_{s} / s\right) .
$$

The main reason for interest in the "edge speed" $\alpha(\lambda)$ is that it enables us to characterize the critical value as

$$
\lambda_{c}=\inf \{\lambda: \alpha(\lambda)>0\}=\sup \{\lambda: \alpha(\lambda)<0\} .
$$

We have already explained this result in one survey article (Durrett (1984)), so we will not give all the details again here. The key to the proof is the following coupling result.

(4) If $A=[a, b]$ and $A \subset B$ then on $\left\{\xi_{t}^{A} \neq \varnothing\right\}$ we have

$$
\xi_{t}^{A}=\xi_{t}^{B} \cap\left[l_{t}^{A}, r_{t}^{A}\right]
$$

where $l_{t}^{A}=\inf \xi_{t}^{A}$ and $r_{t}^{A}=\sup \xi_{t}^{A}$.

(4) is proved by checking that every transition preserves this equality. Applying (4) with $A=\{0\}, B=\{0,-1,-2, \ldots\}$, and letting $r_{t}^{0}=r_{t}^{\{0\}}$, $l_{t}^{0}=l_{t}^{\{0\}}$ we see that if $\alpha(\lambda)<0$ then on $\Omega_{\infty}=\left\{\xi_{t}^{0} \neq \varnothing\right.$ for all $\left.t\right\}$ we have $r_{t}^{0} \leq r_{t} \rightarrow-\infty$ and by symmetry $l_{t}^{0} \rightarrow \infty$, a contradiction, which proves that $\Omega_{\infty}$ has probability 0 .

The converse is more difficult to prove but the result should be intuitive: if $\alpha(\lambda)>0$ then an interval has a tendency to grow. (2) and the coupling result (4) imply that if $M$ is large

$$
\mathrm{P}\left(r_{t}^{[-M, M]}>l_{t}^{[-M, M]} \text { for all } t\right)>0,
$$

so the contact process has positive probability of surviving starting from $[-M, M]$ and hence also when it starts from $\{0\}$. The tricky part of the proof of (3) is to show the second equality, i.e., to rule out the possibility that $\alpha(\lambda)=0$ on an interval.

In the last two paragraphs we have essentially proved the theorem in $d=1$. Using (4) with $A=\{0\}$ and $B=Z$ gives

$$
\xi_{t}^{0}=\xi_{t}^{Z} \cap\left[l_{t}^{0}, r_{t}^{0}\right] \text { on }\left\{\xi_{t}^{0} \neq \varnothing\right\}
$$


and again with $A=\{0\}$ and $B=(-\infty, 0]$ gives

$$
r_{t}^{0}=r_{t} \text { on }\left\{\xi_{t}^{0} \neq \varnothing\right\} \text {. }
$$

Combining the last result with (2) and using symmetry shows that on $\Omega_{\infty}=$ $\left\{\xi_{t}^{0} \neq \varnothing\right.$ for all $\left.t\right\}$ we have with probability one

$$
r_{t}^{0} / t \rightarrow \alpha(\lambda) \text { and } l_{t}^{0} / t \rightarrow-\alpha(\lambda),
$$

so the conclusion of $(1)$ holds with $D=[-\alpha(\lambda), \alpha(\lambda)]$.

In $d>1$ things are much more difficult because we do not have a good way of characterizing $\lambda_{c}$ and the coupling result (4) breaks down. The first result in $d>1$ was proved by Durrett and Griffeath (1982) who showed that the result was valid for $\lambda>\lambda_{c}(Z)$, the critical value for the contact process on $Z$. Looking at that paper now (it is 18 journal pages of computations with lots of details left to the reader) I can sympathize with the referee who read the first twelve pages of the preprint and then skipped to the end to inform us that we had misspelled his name in the list of references. [In a daze, he signed his report, so his identity is not a conjecture.]

The basic ingredient which goes into the proof is, as the reader can probably guess by now, subadditivity of the first-infection times $t(x)=\inf \left\{t: x \in \xi_{t}^{0}\right\}$. As in $\S 3$ these times can be $\infty$ but if we condition on $\Omega_{\infty}=\left\{\xi_{t}^{0} \neq \varnothing\right.$ for all $\left.t\right\}$ they are all finite. That (as the old joke goes) is the good news. The bad news is that we have to find a way to define $t(x, y)$ so that subadditivity holds (or almost holds) and it has the same distribution as $t(y-x)$ conditioned on $\Omega_{\infty}$.

If $|x|=n$ the solution is to wait until $\xi_{t}^{0}$ gets within $n^{1 / 2}$ of $x$ and then pick a particle in $\xi_{t}^{0} \cap\left\{z:|x-z| \leq n^{1 / 2}\right\}$. If the particle we picked has offspring at all later times then we let $t(x, y)$ be the time lag until one of its offspring reaches $y$. If the family line of the particle we picked dies out, we wait until the last of its offspring dies (and if necessary until the process reenters $\left.\left\{z:|x-z| \leq n^{1 / 2}\right\}\right)$ and then pick another particle. Each time we pick a particle we have a positive probability of finding a process which lives forever so we will eventually find a family line which lives forever. When we do this we let $t(x, y)$ be the time lag from the choice of the particle who starts the family which lives forever until $y$ is infected by one of his descendants.

The construction in the last paragraph gives a family of random variables $t(x, y)$ which is not quite subadditive (we have left out the unsuccessful attempts), but close enough to use Richardson's (1973) result to conclude the existence of radial limits for all $x \in Z^{d}$ :

$$
t(0, k x) / k \rightarrow c(x) \quad \text { on }\left\{\xi_{t}^{0} \neq \varnothing \text { for all } t\right\} .
$$

As before if we extend the first-passage times to all $x \in R^{d}$ it is easy to show that the last result remains valid. This only takes care of the first-infection times, but there is enough muscle in the proof to show that if $\varepsilon>0$ then with high probability $\xi_{t}^{0}$ contains $\xi_{t}^{Z} \cap\{x: t(x) \leq(1-\varepsilon) t\}$. To do this one uses embedded one-dimensional processes which come from restricting $\xi_{t}$ to a line and the coupling result (4) above. 
The summary above is admittedly a little sketchy but giving more details would be worse. Fortunately things have been arranged so that no one will ever have to read the paper in question. Durrett and Griffeath (1982) proved a general "shape theorem" that other people can use. We will only state the result for the contact process. See the paper for precise assumptions. Let $t(x)$ and $\tau^{0}$ be as above. Let $H_{t}=\{x: t(x) \leq t\}$ and $K_{t}=\left\{x: \xi_{t}^{0}(x)=\xi_{t}^{1}(x)\right\}$, where $\xi_{t}^{1}$ denotes the process starting from $\xi_{t}^{1}=Z^{d}$ and $\xi_{t}^{i}(x)=1$ if $x \in \xi_{t}^{i}$ and 0 otherwise. Here $H_{t}$ stands for the set of points hit by time $t$ and $K_{t}$ is the set where the two processes are "coupled".

(6) Suppose $\mathrm{P}\left(\tau^{0}=\infty\right)>0$ and there are constants $C, \gamma, \delta \in(0, \infty)$ so that

$$
\mathrm{P}\left(t<\tau^{0}<\infty\right) \leq C e^{-\gamma t},
$$

and if $|x|<\delta t$ then

$$
\mathrm{P}\left(t(x)>t, \tau^{0}=\infty\right) \leq C e^{-\gamma t} .
$$

Then there is a convex set $D$ so that for any $\varepsilon>0$ we have

$$
(1-\varepsilon) t D \cap Z^{d} \subset H_{t} \subset(1+\varepsilon) t D
$$

for all $t$ sufficiently large on $\left\{\tau^{0}=\infty\right\}$. If in addition

$$
\mathrm{P}\left(x \notin K_{t}, \tau^{0}=\infty\right) \leq C e^{-\gamma t}
$$

for $|x|<\delta t$ then for any $\varepsilon>0$ we have

$$
(1-\varepsilon) t D \cap Z^{d} \subset H_{t} \cap K_{t} \subset(1+\varepsilon) t D
$$

for all $t$ sufficiently large on $\left\{\tau^{0}=\infty\right\}$.

The hypotheses in this result are quite strong but (a) they appear as lemmas in every "shape theorem" that I know of, and (b) it is widely believed that they hold whenever $\lambda>\lambda_{c}$. In any case checking them is much less work than repeating the proof. In (1987) Durrett and Schonmann used the last result to improve what is known about the contact process in $d$ dimensions. They showed that by using the renormalized bond construction of Durrett (1984) one could avoid using the coupling result (4) in the study of the onedimensional contact process. This observation allowed them to (i) generalize the results about the contact process to a class of finite range systems (i.e. the birth and death rate at $x$ may depend on the state of $x-L, \ldots, x+R$ where $L$ and $R$ are finite) and (ii) show that for any $L$ the shape theorem holds for all $\lambda>\lambda_{c}\left(Z \times\{-L, \ldots, L\}^{d-1}\right)$, the critical value for the contact process on the indicated set. As $L \uparrow \infty$ the right-hand side decreases to a limit $\lambda_{c}^{*}$ which is the constant in (1). Presumably $\lambda_{c}^{*}=\lambda_{c}$ but this is a difficult open problem. To see that there is something to prove here observe that $\lambda_{c}(\{-L, \ldots L\})=\infty$ but $\lambda_{c}(Z)<\infty$.

NOTES. Durrett and Schonmann (1987) can be consulted for a more complete account of the results given above.

5. Crabgrass. In this process the state at time $t$ is $\xi_{t} \subset Z^{d} / M=$ $\left\{z / M: z \in Z^{d}\right\}$ and $M$ is a large integer. Think of a lawn which consists of a lot of plants with a small spacing between them and forget about the fact 
that there is probably more than one type of weed. With this (and the contact process in mind), we say two points $x$ and $y$ are neighbors if $\|x-y\|_{\infty} \leq 1$, and formulate the dynamics as follows:

If $x \in \xi_{t}$ then $\mathrm{P}\left(x \notin \xi_{t+s} \mid \xi_{t}\right)=s+o(s)$.

If $x \notin \xi_{t}$ then

$$
\mathrm{P}\left(x \in \xi_{t+s} \mid \xi_{t}\right)=\beta s \text { (\# of occupied neighbors) } / v(M)+o(s),
$$

where the $o(s)$ was explained in the last section, and $v(M)=(2 M+1)^{d}-1=$ the number of neighbors a point has. ( $v$ is for volume.)

The normalization above is chosen so that the birth rate from an isolated particle is $\beta$, and hence the critical value

$$
\beta_{c}(M)=\inf \left\{\beta: \mathrm{P}\left(\xi_{t}^{0} \neq \varnothing \text { for all } t\right)>0\right\}
$$

satisfies $\beta_{c}(M) \geq 1$. At first glance, increasing the range of the interaction makes the process more complicated, but in fact as $M \rightarrow \infty$ things get much simpler: $\beta_{c}(M) \rightarrow 1$ and if we fix $\beta>1$ then

$$
\mathrm{P}\left(\xi_{t}^{0} \neq \varnothing \text { for all } t\right) \rightarrow(\beta-1) / \beta \text {. }
$$

The right-hand side is the probability of survival for a branching processa system in which particles die at rate 1 , reproduce at rate $\beta$, and are not limited by the restriction of at most one particle per site.

Intuitively the results above say that the contact process behaves like the branching process when $M$ is large. This is clear when $\xi_{t}^{0}$ is small. The difficulty in proving this is that the number of particles in a branching process with $\beta>1$ grows like $\exp ((\beta-1) t)$ so the contact process begins to notice that it is not a branching process when $t$ is about $C_{d}(\log M) /(\beta-1)$, and in the last two statements we are letting $t \rightarrow \infty$ before $M \rightarrow \infty$. This problem is not too difficult to remedy, but the solution relies on a number of facts about the behavior of branching processes, so we will content ourselves with stating the result.

(1) As $M \rightarrow \infty$ we have

$$
\beta_{c}(M) \approx \begin{cases}1+C / M^{2 / 3} & d=1 \\ 1+C(\log M) / M^{2} & d=2 \\ 1+C / M^{d} & d \geq 3\end{cases}
$$

where $\approx$ means that if $C$ is small (large) then the right-hand side is a lower (upper) bound on $\beta_{c}(M)$.

To explain the result we note that $1+C / M^{d}$ is a trivial lower bound which holds in any dimension - that much birth rate is wasted by particles giving birth on top of their own offspring. With this in mind the result says that the trivial bound is correct in $d \geq 3$, the wrong power in $d=1$, and needs logarithmic corrections in $d=2$. The structure of this result illustrates the concept of a "critical dimension $d_{c}$ " which is one of the most tantalizing open problems about percolation and interacting particle systems. When $d>d_{c}$ (reputed to be 6 for percolation and 4 for the contact process) the behavior of the system is "trivial". For percolation (or the contact process) this means that the percolation probability goes to 0 like $\left(p-p_{c}\right)^{\beta}$ and: $\beta=1$ when 
$d>d_{c}$, there are logarithmic corrections when $d=d_{c}$, and when $d<d_{c} \beta$ is an interesting number $<1$. (For percolation in $d=2, \beta$ is conjectured to be 5/36.) At first glance (1) above might seem to contradict physicists' claims that $d_{c}=4$, but in actuality it is support for their viewpoint. Critical values for the Ising model (which has $d_{c}=4$ ) satisfy a result like (1).

A complete explanation of the last paragraph is beyond the scope of this article and will not be attempted here. Returning to our main subject, our next point is to observe that if we keep $\beta$ and $t$ fixed as $M \rightarrow \infty$, we get a situation which is easy to analyze but which still says something interesting about the time evolution of the process and the asymptotic behavior of an associated nonlinear equation. Consider a sequence of initial states in which sites are independently designated as occupied or vacant and with $\mathrm{P}(x \in$ $\left.\xi_{0}^{M}\right) \rightarrow u(x, 0)$ uniformly on compact sets. (Here the superscript indicates the lattice spacing, not the initial configuration). In this case it turns out that $u_{M}(x, t)=\mathrm{P}\left(x \in \xi_{t}^{M}\right)$ converges uniformly on compact sets to a limit $u(x, t)$ which satisfies

$$
\partial u / \partial t=-u+\beta(1-u)(u * \psi)
$$

where

$$
(u * \psi)(x)=\int u(y) \psi(x-y) d y
$$

and

$$
\psi(y)=(1 / 2)^{d} \quad \text { if }\|y\|_{\infty} \leq 1
$$

The last result is easy to prove and the proof illustrates an important concept called "duality" for interacting particle systems, so we give the proof here. We begin by observing that the graphical representation used to construct the contact process in the last section can be adapted to construct the crabgrass process. For each $x$ we have a rate 1 Poisson process $\left\{T_{n}^{x}, n=\right.$ $1,2, \ldots\}$ of $\delta$ 's which kill a particle at $x$ if one is present, and for each pair of points $x$ and $y$ with $\|x-y\|_{\infty} \leq 1$ we have a rate $\beta / v(M)$ Poisson process $\left\{T_{n}^{x, y}, n=1,2, \ldots\right\}$ of arrows from $x$ to $y$ which cause a birth at $y$ if $x$ is occupied and $y$ is vacant.

Given the graphical representation we can define $(x, 0) \rightarrow(y, t)$ as we did in the last section and let

$$
\xi_{t}^{A}=\{y: \text { there is an } x \text { in } A \text { so that }(x, 0) \rightarrow(y, t)\} .
$$

(here and in what follows superscripts $A, B$, or $x$ indicate the initial configuration). When computing $\xi_{t}^{A}$ on an actual realization it is easier to work backwards from time $t$ to time 0 and this leads naturally to the definition of a dual process: if $s \leq t$ let

$$
\zeta_{s}^{B}=\{x:(x, t-s) \rightarrow(y, t) \text { for some } y \in B\} .
$$

From this definition it should be clear that

$$
\xi_{t}^{A} \cap B=\varnothing \quad \text { if and only if } \zeta_{t}^{B} \cap A=\varnothing .
$$

A little more thought reveals that if we change time $\hat{s}=t-s$ and reverse the direction of the arrows in the graphical representation then $\zeta_{s}^{B}$ is the set of 
points at time $\hat{s}$ which can be reached by starting from $B$ at time $\hat{0}$. The new graphical representation is made from the same types of Poisson processes as the old one, so $\varsigma_{s}^{B} s \leq t$ has the same distribution as $\xi_{s}^{B} s \leq t$ and the relation (3) can be written as

$$
\mathrm{P}\left(\xi_{t}^{A} \cap B=\varnothing\right)=\mathrm{P}\left(\xi_{t}^{B} \cap A=\varnothing\right) .
$$

The "duality equation" (4) is the key to the result about $u_{M}(x, t)=\mathrm{P}(x \in$ $\left.\xi_{t}^{M}\right)$ quoted above. The first step is to prove that $u_{M}(x, t)$ has a limit. To do this we observe that if we let $A=\xi_{0}^{M}$ and $B=\{x\}$ in the duality equation, we get

$$
\mathrm{P}\left(x \notin \xi_{t}^{M}\right)=\mathrm{P}\left(\xi_{t}^{x} \cap \xi_{0}^{M}=\varnothing\right),
$$

and that as $M \rightarrow \infty, \xi_{t}^{x}$ converges in distribution to a branching random walk $Z_{t}^{x}$ in which particles die at rate 1 , and at rate $\beta$ give birth to new particles which are displaced from their parents by a random amount which has a uniform distribution on $[-1,1]^{d}$. To prove the last claim we observe that if we ignore deaths and births lost to occupied sites then the process would behave like a branching process in which births occur at rate $\beta$ and hence

$$
\mathrm{E}\left(\sup _{s \leq t}\left|\xi_{s}^{x}\right|\right) \leq e^{\beta t}
$$

the right-hand side being the expected number of particles at time $t$ in the process with no deaths. Using Markov's inequality with the last result shows

$$
\mathrm{P}\left(\sup _{s \leq t}\left|\xi_{s}^{x}\right| \geq M^{1 / 3}\right) \rightarrow 0 \text {. }
$$

Now if $\xi_{t}^{x}$ always contained $M^{1 / 3}$ particles and if by some unfortunate accident $\xi_{t}^{x}$ was contained in some cube of side 1 , then each particle would be giving birth at rate $\beta$ and a newly created particle would land on another particle with probability $M^{1 / 3} / v(M)$. Even under these extreme conditions simple results about the Poisson process imply that the probability of some particle landing on another one is less than

$$
\beta t \cdot M^{1 / 3} \cdot M^{1 / 3} / v(M) \rightarrow 0
$$

as $M \rightarrow \infty$.

Combining (5) with the fact that $\mathrm{P}\left(y \in \xi_{0}^{M}\right) \rightarrow u(x, 0)$ uniformly on compact sets shows

$$
\mathrm{P}\left(x \notin \xi_{t}^{M}\right) \rightarrow \mathrm{E}\left[\prod_{y \in Z_{t}^{x}}(1-u(y, 0))\right]
$$

To see that the limit satisfies the indicated equation we observe that considering what can happen in $[t, t+\delta]$ and letting $\delta \rightarrow 0$ gives

$$
\frac{d}{d t} \mathrm{P}\left(x \in \xi_{t}^{M}\right)=-\mathrm{P}\left(x \in \xi_{t}^{M}\right)+(\beta / v(M)) \cdot \sum_{y \sim x} \mathrm{P}\left(x \notin \xi_{t}^{M}, y \in \xi_{t}^{M}\right)
$$

where " $y \sim x$ " indicates that the sum is over all the $y$ which are neighbors of $x$. Now $v(M) \approx(2 M+1)^{d}$ so if we can replace the probability in the sum by 
$\mathrm{P}\left(x \notin \xi_{t}^{M}\right) \mathrm{P}\left(y \in \xi_{t}^{M}\right)$ (i.e., in the limit the two events are independent) then the sum will approach

$$
(1-u) \int u(y) \psi(x-y) d y
$$

where

$$
\psi(z)=(1 / 2)^{d} \text { if }\|z\|_{\infty} \leq 1
$$

and we will have shown that (2) holds.

To show that the two events are asymptotically independent we observe that by duality

$$
\mathrm{P}\left(x \notin \xi_{t}^{M}, y \in \xi_{t}^{M}\right)=\mathrm{P}\left(\xi_{t}^{x} \cap \xi_{0}^{M}=\varnothing, \xi_{t}^{y} \cap \xi_{0}^{M} \neq \varnothing\right)
$$

and an extension of the reasoning used above shows that the probability a particle in $\xi_{t}^{x}$ will give birth to a particle which lands on $\xi_{t}^{y}$ goes to 0 , so in the limit $\xi_{t}^{x}$ and $\xi_{t}^{y}$ behave like two independent branching processes.

The result in (2) is useful because it tells us something concrete about the time evolution of the process when $M$ is large, and although the information is not very explicit, it is much better than what we know about the contact process. It is interesting to note that by using (6) and some facts about branching random walks we can show that there is a convex set $D$ so that starting from compactly supported nonzero initial data

$$
u(x, t) \approx \begin{cases}(\beta-1) / \beta & \text { if } x \in(1-\varepsilon) t D \\ 0 & \text { if } x \notin(1+\varepsilon) t D .\end{cases}
$$

We begin by recalling some facts about branching random walks. Let $Z_{t}^{0}$ be a branching random walk starting from a single particle at 0 and let $Z_{t}^{0}(A)$ be the number of particles in $A$ at time $t$. It is easy to compute the expected value of $Z_{t}^{0}(A)$. Let $S_{t}$ be a continuous-time random walk which takes steps at rate $\beta$; i.e., the probability it stays in one place for $t$ units of time is $\exp (-\beta t)$ and when it leaves $x$ it jumps to a new place $x+y$, where $y$ is chosen according to a uniform distribution on $[-1,1]^{d}$. In terms of $S_{t}$ the expected value can be written as

$$
\mathrm{E}\left(Z_{t}(A)\right)=e^{(\beta-1) t} \mathrm{P}\left(S_{t} \in A\right) .
$$

The first factor gives the expected number of particles in $Z_{t}^{0}$ so the equation can be interpreted as saying if we pick a particle at random from those in $Z_{t}^{0}$ its location has distribution $S_{t}$.

(8) relates the mean number of particles in $A$ to a random walk probability and since the first factor grows exponentially it brings us to a question of large deviations for the random walk. By now it is well known that there is a function $h(x)$ so that if $B$ is a closed set so that the Lebesgue measure of $\partial B$ is 0 then as $t \rightarrow \infty$

$$
(1 / t) \log \mathrm{P}\left(S_{t} \in t B\right) \rightarrow \sup _{x \in B} h(x),
$$

where $h(x)<0$ for $x \neq 0$. In the case under consideration $h$ can be described as:

$$
h(x)=\inf _{\theta}(-x \cdot \theta-\beta(1-k(\theta)))
$$


where

$$
k(\theta)=\prod_{i=1}^{d} \sinh \left(\theta_{i}\right)=\int_{\|y\|_{\infty} \leq 1} \ldots \int^{\theta \cdot y} d y / 2^{d} .
$$

The last result takes a while to prove but it is not hard to see why it is the answer. $k(\theta)$ is the "moment generating function" of the distribution of a single step. The random walk takes a Poisson mean $\beta t$ number of steps in time $t$ so

$$
\mathrm{E}\left(\exp \left(\theta \cdot S_{t}\right)\right)=\sum_{n=0}^{\infty} e^{-\beta t} \frac{(\beta t)^{n}}{n !} k(\theta)^{n}=\exp (-\beta t(1-k(\theta))) .
$$

If $x \cdot \theta>0$ then using Markov's inequality on the last quantity gives

$$
e^{t x \cdot \theta} \mathrm{P}\left(S_{t} \cdot \theta \geq t x \cdot \theta\right) \leq \mathrm{E}\left(\exp \left(S_{t} \cdot \theta\right)\right) .
$$

Taking logs of both sides and dividing by $t$ gives

$$
(1 / t) \log \mathrm{P}\left(S_{t} \cdot \theta \geq t x \cdot \theta\right) \leq-x \cdot \theta+\beta(1-k(\theta)) .
$$

This last result holds for all $\theta$ with $x \cdot \theta>0$. To get the best possible bound from this, we minimize over $\theta$ (and observe that this will occur when $x \cdot \theta>0$ ), and the result is the expression in (10).

Having derived a formula for $h(x)$ we will now ignore it for the rest of the proof of (7). (9) gives us

$$
(1 / t) \log \mathrm{E}\left(Z_{t}(t B)\right) \rightarrow(\beta-1)+\sup _{x \in B} h(x),
$$

so we guess that (7) will hold with

$$
D=\{x:(\beta-1)+h(x) \geq 0\} .
$$

Since

$$
\mathrm{P}\left(Z_{t}^{0}(t B) \geq 1\right) \leq \mathrm{E}\left(Z_{t}^{0}(t B)\right),
$$

letting $B=[(1+\varepsilon) D]^{c}$ shows us that if $\varepsilon>0$ then with high probability $Z_{t}$ will have no points outside of $t(1+\varepsilon) D$. If $u(\cdot, 0)$ has support in $K$ and $x \notin D$ then using (6) and the last observation gives

$$
1-u(t x, t)=\mathrm{E}\left[\prod_{y \in Z_{t}^{x t}}(1-u(y, t))\right] \geq \mathrm{P}\left(Z_{t}^{x t} \cap K=\varnothing\right) \rightarrow 1
$$

as $t \rightarrow \infty$.

The last conclusion proves half of (7). To prove the other half let $\Omega_{\infty}=$ $\left\{\left|Z_{t}^{0}\right|>0\right.$ for all $\left.t\right\}$, and observe that $\mathrm{P}\left(\Omega_{\infty}\right)=(\beta-1) / \beta$. Then a result of Biggins (1978) shows that if $(\beta-1)+h(x)>0$ then except for a set with probability 0 we have

$$
(1 / t) \log Z_{t}^{0}\left(x t+(-1,1)^{d}\right) \rightarrow(\beta-1)+h(x)
$$

on $\Omega_{\infty}$ as $t \rightarrow \infty$. The last result holds if $(-1,1)^{d}$ is replaced by any open set. Using this it is easy to see that if $x$ is in the interior of the set $D$ defined 
above then when $t$ is large either $Z_{t}^{x t}=\varnothing$ or $(1 / t) \log Z_{t}^{x t}(\{y: u(y, 0)>0\}) \approx$ $(\beta-1)+h(x)$, so

$$
1-u(t x, t)=\mathrm{E}\left[\prod_{y \in Z_{t}^{x t}}(1-u(y, 0))\right]=\mathrm{P}\left(Z_{t}^{x t}=\varnothing\right)+o(1)
$$

where $o(1)$ stands for a term which goes to 0 as $M \rightarrow \infty$, and this proves the second half of (7).

NOTES. The proof of (1) will appear in Bramson, Durrett, and Swindle (198?). (7) and related questions are the subject of Swindle's dissertation and of the author's current research.

Appendix. In this section we will explain some of the unfamiliar words and results used in the paper. Most of these terms are concepts from measure theory which have been renamed. The stage on which all the action takes place is called a probability space. It is usually denoted $(\Omega, \mathcal{F}, P)$ where $\Omega$ is a set, $\mathcal{F}$ is a $\sigma$-field of subsets of $\Omega$, and $P$ is a probability measure; as in this article, it almost never explicitly appears.

Sets $A \in \mathcal{F}$ are called events and measurable functions $X(\omega)$ are called random variables. When we integrate a random variable over the entire space, i.e.

$$
\int_{\Omega} X(\omega) d P(\omega)
$$

the result is called the expected value of $X$ and denoted $\mathrm{E}(X)$. The next two results will give the reader practice with this notation and introduce two more. Observe that Fubini's theorem implies:

(1) If $X \geq 0$ then

$$
\begin{aligned}
\mathrm{EX} & =\mathrm{E}\left(\int_{0}^{\infty} 1_{\{\omega: X(\omega) \geq x\}} d x\right) \\
& =\int_{0}^{\infty} \mathrm{P}(X \geq x) d x,
\end{aligned}
$$

where $1_{A}(\omega)=1$ if $\Omega \in A$ and 0 otherwise (it is " 1 on $A$ ").

Common sense gives us Markov's inequality:

(2) If $X \geq 0$ and $f \geq 0$ is nondecreasing on $[0, \infty)$ then

$$
\mathrm{E} f(X) \geq \mathrm{E}\left(f(X) 1_{\{X \geq x\}}\right) \geq f(x) \mathrm{P}(X \geq x),
$$

so

$$
\mathrm{P}(X \geq x) \leq \mathrm{E}(f(X)) / f(x) .
$$

Last but not least, we have the notion which makes probability different from measure theory: independence. Two events $A$ and $B$ are said to be independent if $\mathrm{P}(A \cap B)=\mathrm{P}(A) \mathrm{P}(B)$. Two random variables are called independent if for all $x$ and $y$

$$
\mathrm{P}(X \leq x, Y \leq y)=\mathrm{P}(X \leq x) \mathrm{P}(Y \leq y)
$$

The fundamental fact about independent random variables is the strong law of large numbers. Let $X_{1}, X_{2}, \ldots$ be a sequence of independent and identically 
distributed random variables, i.e., they all have the same distribution function $F(x)=\mathrm{P}\left(X_{i} \leq x\right)$. If $\mathrm{E}|X|<\infty$ and $\mathrm{E} X=\mu$ then with probability one (or, more formally, for almost every $\omega$ in the underlying space $\Omega$ )

$$
\left(X_{1}+\cdots+X_{n}\right) / n \rightarrow \mu
$$

as $n \rightarrow \infty$. This is the "fundamental theorem of statistics": as $n \rightarrow \infty$, the average of the first $n$ observations approaches the mean $\mu$ (another word for expected value).

ACKNOWLEDGMENTS. To keep the list of references short, we have listed only the papers referred to in the text and the most recent surveys for each of the areas we have touched on. This approach robs some of the original contributors (most notably J. M. Hammersley, R. T. Smythe and J. C. Wierman, T. E. Harris, N. T. J. Bailey, D. Mollison, and K. Kuulasmaa) of the credit they deserve. We apologize to anyone who feels slighted.

Finally I would like to thank my colleagues, friends, and co-authors without whose help this would not have been possible: Maury Bramson, Lincoln and Jennifer Chayes, Ted Cox, David Griffeath, Ted Harris, Tom Liggett, Harry Kesten, and Roberto Schonmann.

\section{REFERENCES}

M. Aizenman, J. T. Chayes, L. Chayes, J. Frohlich, and L. Russo (1983) On a sharp transition from area law to perimeter law in a system of random surfaces, Comm. Math. Phys. 92, $19-69$.

J. D. Biggins (1978) The asymptotic shape of the branching random walk, Adv. Appl. Prob. 1.0, 62-84.

M. Bramson, R. Durrett, and G. Swindle (198?) Statistical mechanics of crabgrass, Ann. Prob., to appear.

J. T. Cox and R. Durrett (1981) Some limit theorems for percolation processes with necessary and sufficient conditions, Ann. Prob. 9, 583-603.

Appl., to appear.

(198?) Limit theorems for the spread of epidemics and forest fires, Stoch. Proc.

R. Durrett (1980) On the growth of one dimensional contact processes, Ann. Prob. 8, 890-907.

_, (1984) Oriented percolation in two dimensions, Ann. Prob. 12, 999-1040.

(1985) Particle systems, random media, and large deviations, Contemp. Math., Vol.

41, Amer. Math. Soc., Providence, R. I.

R. Durrett and D. Griffeath (1982) Contact processes in several dimensions, Z. Wahrsch. Verw. Gebiete 59, 535-552.

, (1983) The supercritical contact process on Z, Ann. Prob. 11, 1-15.

R. Durrett and T. Liggett (1981) The shape of the limit set in Richardson's model, Ann. Prob. 9, 186-193.

R. Durrett and R. H. Schonmann (1987) Stochastic growth models, Percolation Theory and Ergodic Theory of Infinite Particle Systems, I.M.A. Volumes in Math. and its Applications, Vol. 8, Springer-Verlag.

H. Kesten (1982) Percolation theory for mathematicians, Birkhäuser, Boston. , (1986) Aspects of first passage percolation, Lecture Notes in Math., vol. 1180, Springer-Verlag, Berlin and New York.

, (1987a) Surfaces with minimal random weights and maximal flows: $A$ higher dimensional version of first passage percolation, Illinois J. Math. 31, 99-166.

, (1987b) Percolation theory and first passage percolation, Ann. Prob. 15, 1231-1271.

J. F. C. Kingman (1973) Subadditive ergodic theory, Ann. Prob. 1, 883-909.

T. Liggett (1985a) An improved subadditive ergodic theorem, Ann. Prob. 13, 1279-1285. 
(1985b) Interacting particle systems, Springer-Verlag, New York.

D. Richardson (1973) Random growth in a tesselation, Proc. Cambridge Philos. Soc. 74, 515-528.

L. Russo (1981) On the critical percolation probabilities, Z. Wahrsch. Verw. Gebiete 56, 229-237.

R. T. Smythe and J. C. Wierman (1978) First passage percolation on the square lattice, Lecture Notes in Math., vol. 671, Springer-Verlag, Berlin and New York.

P. Tautu (1986) Stochastic spatial processes, Lecture Notes in Math., vol. 1212, SpringerVerlag, Berlin and New York.

J. C. Wierman (1981) Bond percolation on honeycomb and triangular lattices, Adv. Appl. Prob. 13, 293-313.

, (1982) Percolation theory, Ann. Prob. 10, 509-524.

Department of Mathematics, Cornell University, Ithaca, NeW York, 14853 
